\title{
Ferulic acid ameliorates nerve injury induced by cerebral ischemia in rats
}

\author{
LEIMING ZHANG, HONGSHENG WANG, TIAN WANG, NA JIANG, \\ PENGFEI YU, YATING CHONG and FENGHUA FU
}

Department of Pharmacology, School of Pharmacy, Yantai University, Yantai, Shandong 264005, P.R. China

Received March 12, 2014; Accepted October 23, 2014

DOI: 10.3892/etm.2014.2157

\begin{abstract}
This study was designed to investigate the protective effect of ferulic acid (FA) on nerve injury induced by cerebral ischemia. Focal cerebral ischemia was induced by occlusion of the right middle cerebral artery and reperfusion 90 min later in male Sprague-Dawley rats. Daily treatment of the rats with FA was initiated $30 \mathrm{~min}$ after the surgery, and was continued for 7 days. The efficacy of FA against nerve injury was assessed by neurological deficit scores as well as pathohistological observation. The expression levels in the brain and level in the peripheral blood of erythropoietin (EPO) and granulocyte colony-stimulating factor (G-CSF) were analyzed by immunohistochemistry and enzyme-linked immunosorbent assay (ELISA), respectively. The results showed that FA attenuated nerve injury of the hippocampus, significantly ameliorated neurological deficits, and increased EPO but not G-CSF expression in the hippocampus and the peripheral blood of ischemic rats. The findings indicate that FA has certain protective effects on the nerve injury of cerebral ischemia, and suggest that promoting EPO in the brain and peripheral blood may be one of the neuroprotective mechanisms of FA.
\end{abstract}

\section{Introduction}

Stroke is a serious threat to human health, and is recognized as the most important factor resulting in nerve injury $(1,2)$. Evidence suggests that nerve injury occurs following ischemia in susceptible brain regions, such as the hippocampus and the cerebral cortex. Neuronal cell death has been identified by morphological analysis of the ischemic brain, and genetic and biochemical evidence further support the association of neuronal cell death with ischemia $(3,4)$.

Correspondence to: Professor Fenghua Fu, Department of Pharmacology, School of Pharmacy, Yantai University, 30 Qingquan Road, Yantai, Shandong 264005, P.R. China

E-mail: fufenghua@sohu.com

Key words: ferulic acid, erythropoietin, cerebral ischemia, nerve injury
Angelicae sinensis is a traditional Chinese medicinal herb that has long been used to treat ischemic stroke and anemia $(5,6)$. The chemical constituents of the extract of the roots of Angelica sinensis are classified into essential oil- and water-soluble materials (7). Ferulic acid (FA) is the main water-soluble component of the roots of Angelica sinensis. Previous studies have demonstrated that FA is able to enhance hematopoietic progenitor cell activity resulting in accelerated blood cell recovery by stimulating erythropoietin (EPO) and granulocyte colony-stimulating factor (G-CSF) expression $(8,9)$. A further study demonstrated that FA was able to reduce cerebral infarction in a model of transient middle cerebral artery occlusion (10).

EPO and G-CSF were originally recognized as humoral mediators involved in the maturation and proliferation of hematopoietic progenitor cells (11), and their neuroprotective effects were subsequently identified. In vitro and in vivo studies in animal models revealed that exogenously administered EPO and G-CSF are neuroprotective $(12,13)$. Endogenous EPO and G-CSF have also demonstrated beneficial effects in experimental stroke $(14,15)$.

Although FA has been shown to reduce cerebral infarct secondary to transient focal cerebral ischemia, little data is available regarding its neuroprotective effect on nerve injury and the related protective factors EPO and G-CSF. Thus, the aim of this study was to investigate the protective effect of FA on nerve injury induced by cerebral ischemia and whether the cerebroprotective effect of FA is associated with EPO and $\mathrm{G}-\mathrm{CSF}$ induction in the rat brain.

\section{Materials and methods}

Animals and drugs. Male Sprague-Dawley rats weighing $220 \pm 20 \mathrm{~g}$ were obtained from Experimental Animal Center of Shandong University of Chinese Traditional Medicine (Jinan, China). They were kept in air-conditioned rooms (temperature, $23 \pm 2{ }^{\circ} \mathrm{C}$ ) on a $12 \mathrm{~h}$ light-dark cycle, with free access to food and water. Animal experimental procedures were carried out in strict accordance with the guidelines published by the United States National Institutes of Health (NIH Publication no. 85-23, revised 1996) and approved by the ethics committee of Yantai University (Shandong, China). Surgical procedures, stroke induction and animal sacrifice (at the end of the observation period) were performed under 
general anesthesia with intraperitoneal (i.p.) injection of chloral hydrate $(350 \mathrm{mg} / \mathrm{kg})$.

The sodium salt of FA (i.e., sodium ferulate) was obtained from Haikou Qili Pharmaceutical Co., Ltd. (Haikou, China) and edaravone injection was provided by Nanjing Simcere Dongyuan Pharmaceutical Co., Ltd. (Nanjing, China).

Rat cerebral ischemia study protocol. The middle cerebral artery occlusion (MCAO) procedure was carried out according to a previously described method with minor modifications (16). Briefly, rats were anesthetized with $10 \%$ chloral hydrate in $0.9 \% \mathrm{NaCl}(350 \mathrm{mg} / \mathrm{kg}$, i.p.) and placed in a dorsal recumbent position. Under sterile conditions, a ventral neck incision was made and the external carotid artery (ECA) and internal carotid artery (ICA) were exposed and carefully isolated. A nylon monofilament $(40 \mathrm{~mm}$ in length and $0.25 \mathrm{~mm}$ in diameter), its tip rounded by flame-heating, was inserted from the lumen of the ECA to that of the right ICA to occlude the origin of the right MCA. The filament was removed after $1 \mathrm{~h}$. For the sham group, the ECA and ICA underwent the same procedures without occlusion of the MCA. The rats were kept under conditions of controlled temperature $\left(24-25^{\circ} \mathrm{C}\right)$ for the first $24 \mathrm{~h}$ after surgery.

The rats were randomly divided into six groups of 10 rats each. Three groups received different doses of FA (50, 100 and $200 \mathrm{mg} / \mathrm{kg})$, and one group received edaravone $(6 \mathrm{mg} / \mathrm{kg})$ by intravenous injection $30 \mathrm{~min}$ after ischemia, and once a day thereafter for seven consecutive days. The rats in the sham and vehicle-treated group were injected with saline.

Evaluation of neurological deficits. Neurological deficits were evaluated using a modified six-point scoring method (17), by an investigator who was blinded to each experimental group. The damage was graded on a scale of 0-5. The scale is: 0 , no neurological deficits (normal); 1 , failure to extend left forepaw fully (mild); 2 , circling to the left (moderate); 3 , falling to the left (severe); 4, no spontaneous walking with a depressed level of consciousness (very severe); and 5, death.

Hematoxylin and eosin $(H \& E)$ staining. Six rats from each group were selected for H\&E staining. The rats were deeply anesthetized with choral hydrate and pericardially perfused with $0.9 \%$ saline and then with $4 \%$ formaldehyde. The entire brain was embedded in paraffin. Nerve injury in the hippocampus was determined by analysis under a microscope (magnification, x400; Olympus BX41; Olympus, Tokyo, Japan). The damage was evaluated by counting the number of surviving neurons per millimeter length of the hippocampus examined under light microscopy.

Enzyme-linked immunosorbent assay (ELISA). EPO and G-CSF levels in the plasma were measured by ELISA kits (EPO Quantikine ELISA Kit, catalogue no. MEP00B and G-CSF Quantikine ELISA Kit, catalogue no. MCS00; R\&D Systems, Inc., Minneapolis, MN, USA). The rats in each group were sacrificed $24 \mathrm{~h}$ after the final administration of treatment and plasma was collected. Monoclonal antibodies specific for EPO and G-CSF were pre-coated onto microplates. Standards and samples were added to the wells and were incubated for $30 \mathrm{~min}$ at $37^{\circ} \mathrm{C}$. After washing away the unbound substances,
Table I. Effects of FA on neurological deficit scores in rats following MCAO $(n=10)$.

\begin{tabular}{lc}
\hline Group & Neurological deficit scores (median/range) \\
\hline Sham & - \\
Vehicle & $4 / 2$ \\
Edaravone & $2 / 4^{\mathrm{b}}$ \\
FA $(50 \mathrm{mg} / \mathrm{kg})$ & $4 / 4$ \\
FA $(100 \mathrm{mg} / \mathrm{kg})$ & $3 / 4^{\mathrm{a}}$ \\
FA $(200 \mathrm{mg} / \mathrm{kg})$ & $2 / 4^{\mathrm{a}}$ \\
\hline
\end{tabular}

FA, ferulic acid; MCAO, middle cerebral artery occlusion. ${ }^{\mathrm{a}} \mathrm{P}<0.05$; ${ }^{\mathrm{b}} \mathrm{P}<0.01$, compared with the vehicle group.

enzyme-linked polyclonal antibodies specific for EPO and G-CSF were added to the wells and were incubated for $60 \mathrm{~min}$ at $37^{\circ} \mathrm{C}$. After removing any unbound antibodies, substrates were added to the wells and were incubated for $15 \mathrm{~min}$ at $37^{\circ} \mathrm{C}$. The intensities of the colors developed were in proportion to the amount of EPO and G-CSF bound to the wells. The optical density of each well was measured with a scanning multi-well spectrophotometer (SpectraMax M3, Molecular Devices, Sunnyvale, CA, USA) at a wavelength of $450 \mathrm{~nm}$.

Immunohistochemistry assays. After being deeply anesthetized, the rats were transcardially perfused with saline solution, followed by $4 \%$ paraformaldehyde in $0.1 \mathrm{M}$ phosphate-buffered saline (PBS) $24 \mathrm{~h}$ after ischemia. Brains were removed and post-fixed in $4 \%$ paraformaldehyde for $4 \mathrm{~h}$, then transferred into $30 \%$ sucrose solution until the brains sank to the bottom of the container. Coronal sections $(10 \mu \mathrm{m})$ were made using a Leica CM1950S cryostat (Leica Microsystems GmbH, Wetzlar, Germany). Sections were blocked with $3 \%$ normal goat serum (diluted in PBS containing $0.3 \%$ Triton $\mathrm{X}-100$ ) for $1 \mathrm{~h}$ and incubated with rabbit anti-rat polyclonal primary antibodies [antiEPO (BA0843) and anti-G-CSF (BA0746), 1:200, Wuhan Boster Biological Engineering Co., Ltd., Wuhan, China] overnight at $4^{\circ} \mathrm{C}$. After rinsing with PBS, sections were incubated with horseradish peroxidase-conjugated goat anti-rabbit polyclonal IgG (ZDR-5306; Beijing Zhongshan Jinqiao Biological technology Co. Ltd., Beijing, China) as secondary antibodies (1:200) for $2 \mathrm{~h}$ at room temperature. The images from five fields of each ischemic region from six rats in each group were examined using the same brightness and exposure settings. Image-Pro Plus software (Media Cybernetics, Silver Spring, MD, USA) was used to analyze positive expression of EPO or G-CSF in each photograph. The artificial unit of mean optical density (MOD) $x$ total per area (TPA) was employed for measurement in the stereological analysis, which indicated the integrated optical density (IOD) of the positive signal in the stained tissue.

Statistical analysis. Neurological deficit scores between groups were analyzed using a non-parametric test. Quantitative data from the experiments were expressed as mean \pm standard deviation (SD), and significance was determined by one-way 

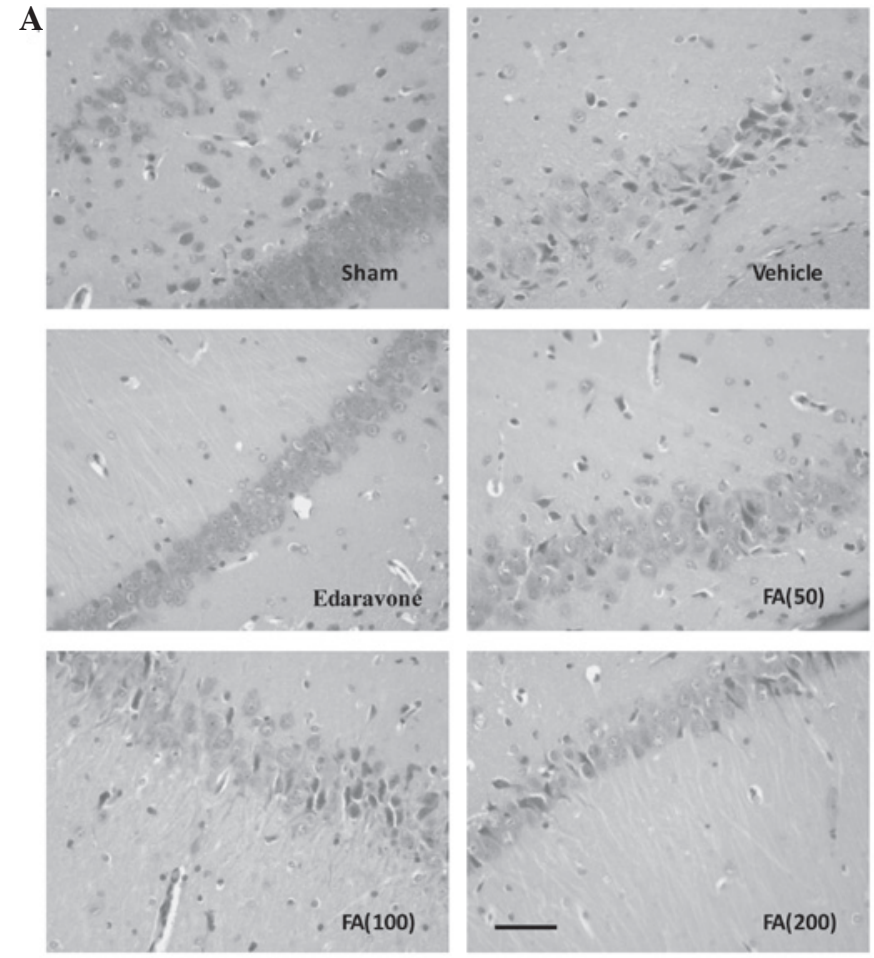

B

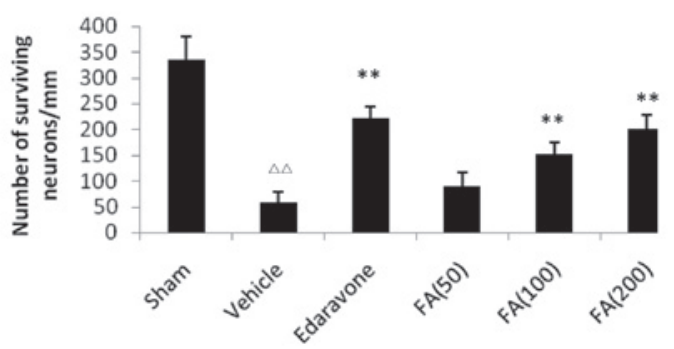

Figure 1. (A) Histopathology in the ischemic region following MCAO. Light microphotographs of the hippocampus with hematoxylin and eosin staining in the sham-treated control group, vehicle-treated ischemia model group, edaravone-treated group and FA-treated groups $(50,100$ and $200 \mathrm{mg} / \mathrm{kg}$ ). (B) Extensively damaged neurons in hippocampus were observed in the ischemia model group and the number of surviving neurons was significantly reduced compared with that in the sham-treated group. FA (at doses of 100 and $200 \mathrm{mg} / \mathrm{kg}$ ) and edaravone treatment increased the number of neurons and numerous normal surviving neurons were observed. Scale bar: $40 \mu \mathrm{m}$; data expressed as mean \pm standard deviation, $n=6 ;{ }^{\Delta} \mathrm{P}<0.01$ vs. sham-treated mice; ${ }^{* *} \mathrm{P}<0.01$ vs. vehicle-treated ischemia model mice.

analysis of variance (ANOVA) followed by Tukey's test. In all cases, differences were considered significant if $\mathrm{P}<0.05$.

\section{Results}

Effects of FA on neurological deficit scores in rats following $M C A O$. On the seventh day after the surgery, the results showed that FA induced a significant reduction in the neurological deficit score compared with that of the rats treated with vehicle. Treatment with FA at doses of 100 and $200 \mathrm{mg} / \mathrm{kg}$ but not $50 \mathrm{mg} / \mathrm{kg}$ significantly reduced the neurological deficit score in a dose-dependent manner in the MCAO model rats. Edaravone treatment, as a positive control, also significantly decreased the neurological deficit score (Table I).

Effects of FA on neuronal damage in the hippocampus of rats following MCAO. Extensively damaged neurons in the hippo-
Table II. Effects of FA on EPO and G-CSF levels in the peripheral blood of rats following MCAO (mean \pm standard deviation; $n=6$ ).

\begin{tabular}{lcc}
\hline Group & EPO $(\mu \mathrm{g} / \mathrm{ml})$ & G-CSF $(\mu \mathrm{g} / \mathrm{ml})$ \\
\hline Sham & $8.32 \pm 2.85$ & $0.39 \pm 0.09$ \\
Vehicle & $8.63 \pm 0.97$ & $0.78 \pm 0.24^{\mathrm{c}}$ \\
Edaravone & $8.62 \pm 2.51$ & $0.75 \pm 0.19$ \\
FA $(50 \mathrm{mg} / \mathrm{kg})$ & $12.68 \pm 3.84^{\mathrm{a}}$ & $0.62 \pm 0.11$ \\
FA $(100 \mathrm{mg} / \mathrm{kg})$ & $13.28 \pm 3.32^{\mathrm{a}}$ & $0.72 \pm 0.12$ \\
FA $(200 \mathrm{mg} / \mathrm{kg})$ & $12.38 \pm 1.91^{\mathrm{b}}$ & $0.60 \pm 0.12$ \\
\hline
\end{tabular}

FA, ferulic acid; EPO, erythropoietin; G-CSF, granulocyte colony-stimulating factor; MCAO, middle cerebral artery occlusion. ${ }^{\mathrm{a}} \mathrm{P}<0.05,{ }^{\mathrm{b}} \mathrm{P}<0.01$, compared with the vehicle group; ${ }^{\mathrm{c}} \mathrm{P}<0.01$, compared with the sham group.

campus were observed and the number of surviving neurons was significantly reduced in the vehicle-treated ischemic rats compared with the sham-treated rats. Neuronal shrinkage and chromatin condensation of nuclei were also observed in the ischemic rats. However, the number of surviving neurons in the FA-treated rats was higher compared with that in the vehicle-treated ischemic rats and numerous surviving neurons were observed in the hippocampus (Fig. 1).

Effects of FA on EPO and G-CSF levels in the peripheral blood of rats following MCAO. FA induced significant enhancements in the EPO level in peripheral blood compared with that in the vehicle-treated rats $(\mathrm{P}<0.05)$. With regard to G-CSF levels, the rats treated with FA showed no significant difference compared with the vehicle-treated rats (Table II).

Effects of FA on EPO and G-CSF expression in the hippocampus of rats following MCAO. The results showed that EPO expression within the infarct region in the vehicle-treated group was significantly increased compared with that of the sham-operated group. Administration of FA induced a significant enhancement in EPO expression levels in a dose-dependent manner $(\mathrm{P}<0.01)$. With regard to G-CSF, cerebral ischemia induced a significant increase in its expression, whereas treatment with FA was not observed to induce a significant difference in the expression of the protein in the infarct region compared with that in the vehicle-treated rats (Fig. 2).

\section{Discussion}

Nerve injury resulting from focal or global cerebral ischemia is a major cause of mortality and disability in the adult population. Neuronal cell death has been observed to occur several days after ischemic insult and predominantly affects sensitive areas of the brain, such as the hippocampus and the cortex $(18,19)$.

The results of the present study demonstrated that neuronal cell death occurred in the ischemic brain primarily in the hippocampal area seven days after ischemia. Treatment with FA significantly reduced the neurological deficit score and 

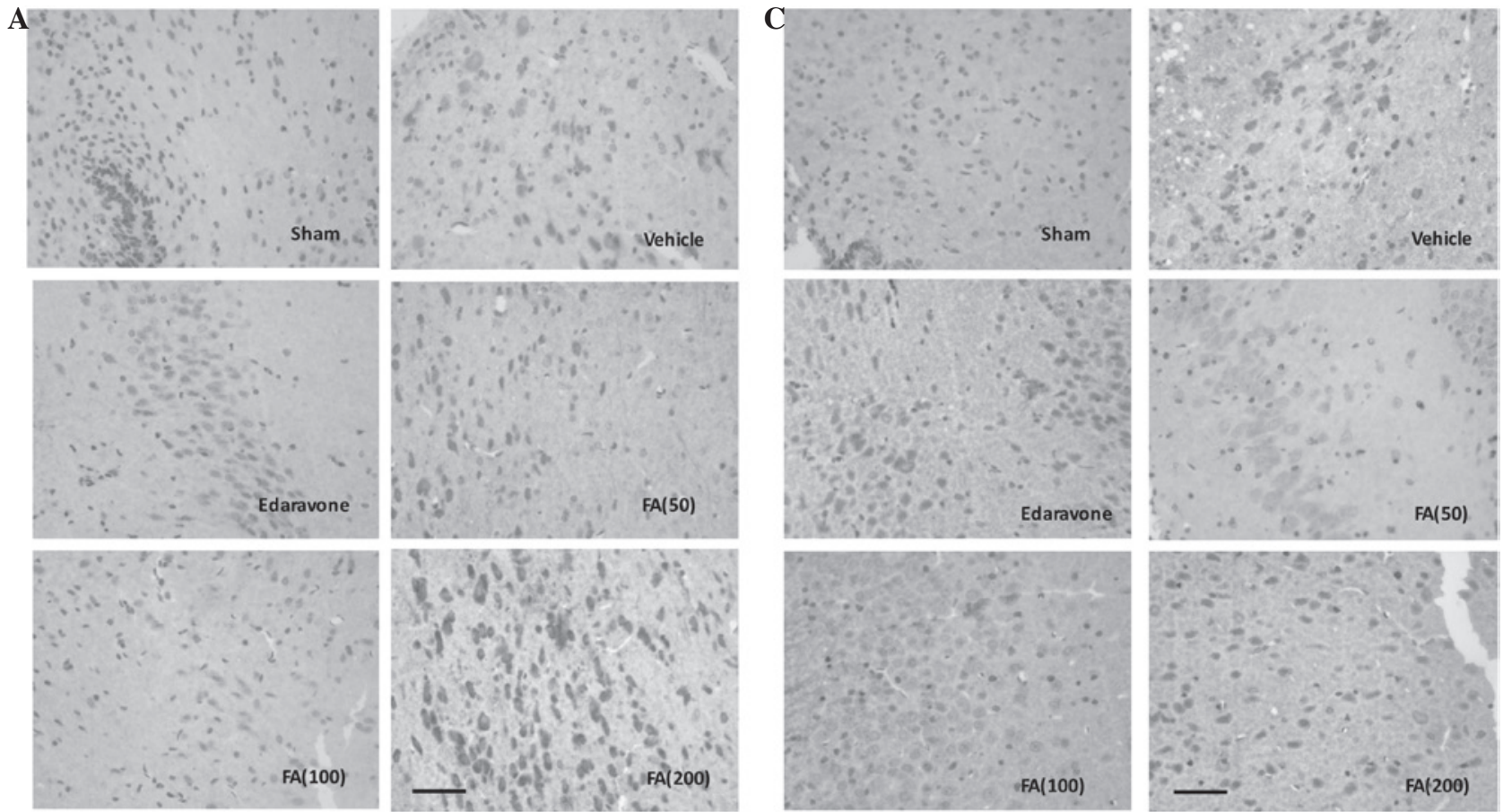

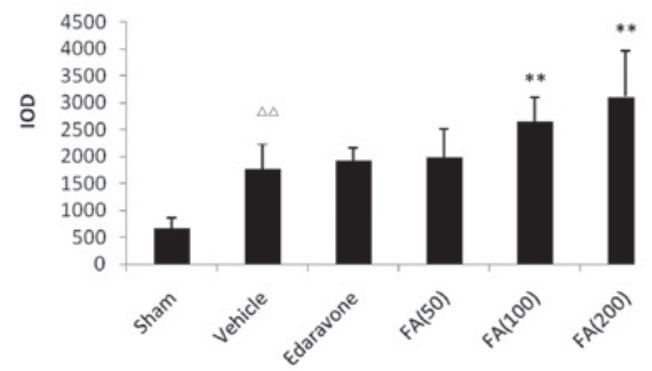

D

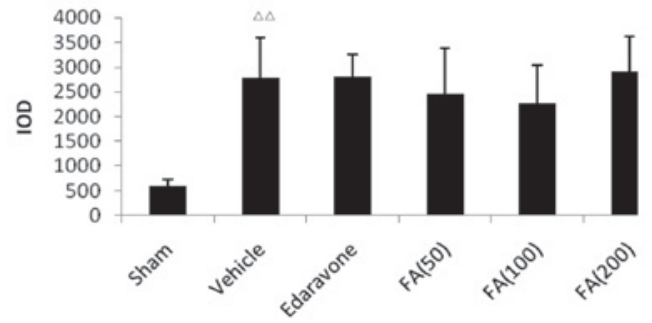

Figure 2. EPO and G-CSF expression as revealed by immunohistochemistry in rats following MCAO and the subsequent administration of different treatments. (A and B) EPO expression within the infarct region of the hippocampus in the vehicle-treated group was significantly increased compared with that of the sham-operated group. Administration of FA induced a significant enhancement in EPO expression in a dose-dependent manner (P<0.01). (C and D) In the case of G-CSF, cerebral ischemia induced a significant increase, while treatment with FA showed no significant difference in the expression of the protein in the infarct region, compared with that in the vehicle-treated rats. Scale bar: $40 \mu \mathrm{m}$; data expressed as mean \pm standard deviation, $\mathrm{n}=6$; ${ }^{\Delta \Delta} \mathrm{P}<0.01 \mathrm{vs}$. shamtreated mice; ${ }^{* *} \mathrm{P}<0.01$ vs. vehicle-treated ischemic mice. EPO, erythropoietin; G-CSF, granulocyte colony-stimulating factor; MCAO, middle cerebral artery occlusion; FA, ferulic acid.

hippocampal neuron damage in a dose-dependent manner in the rats following MCAO. The results indicated that treatment of the rats with FA for seven days following MCAO ameliorated the neuronal damage.

EPO stimulates erythroid cell production, which supports the survival, proliferation and differentiation of erythroid progenitor cells. The sites of expression of the EPO receptor (EPO-R) and the EPO response are in hematopoietic cells and other cell types, including endothelial and neural cells (20). The targeted deletion of EPO or the EPO-R causes mice to lack definitive erythropoiesis and mature erythrocytes (21). In such mice, increased levels of apoptosis in the brain are also observed, which suggests that EPO, in addition to being necessary for the production of mature red blood cells, may also contribute to normal brain development (22). EPO is produced in fetal liver, adult kidney and also in the brain, in astrocytes and neurons; EPO production is induced by hypoxia, and may persist for $24 \mathrm{~h}$ or longer (23).

In the present study, it was observed that FA induced a significant enhancement of the level of EPO expression in the ischemic brain. The results also demonstrated that FA increased the EPO level in peripheral blood; EPO nay be transferred from the blood into the brain through the blood-brain barrier (BBB) (24). Therefore, the increasing levels of EPO in the brain and blood may contribute to the neuroprotective effect of FA.

The hematopoietic factor G-CSF was recently discovered to act as a protective and neurotrophic factor in the brain. Several studies have described the infarct-reducing and recovery-enhancing effects of G-CSF following ischemic stroke (25-27). The main actions of G-CSF are mediated via binding to the G-CSF receptors present on neuronal cells. In the present study, however, treatment with FA for seven days demonstrated no significant effect on the levels of G-CSF in the ischemic brain and peripheral blood. Although the traditional Chinese medicine Angelicae sinensis is commonly used to promote erythropoiesis for the treatment of anemia, and FA has previously been demonstrated to increase the levels of EPO and G-CSF $(8,9)$, the results of the present study suggest that the neuroprotective effect of FA is not associated with G-CSF. 
The findings indicate that FA has certain protective effects against the nerve injury induced by cerebral ischemia, and suggest that the promotion of EPO expression in the ischemic brain and peripheral blood may be one of the neuroprotective mechanisms of FA.

\section{Acknowledgements}

This study was supported by the Project of Shandong Province Higher Educational Science and Technology Program (No. 10LF76), the Foundation for Outstanding Middle-age and Young Scientists (No. BS2011YY061) and Taishan Scholar Project. The authors are grateful to Professor Tongshen Liu for providing technical assistance in the pathological observations.

\section{References}

1. Gorelick PB: Stroke prevention therapy beyond antithrombotics: unifying mechanisms in ischemic stroke pathogenesis and implications for therapy: an invited review. Stroke 33: 862-875, 2002.

2. Fisher M: Advances in stroke 2007: introduction. Stroke 39: 250-251, 2008

3. Honkaniemi J, Massa SM, Breckinridge M and Sharp FR: Global ischemia induces apoptosis-associated genes in hippocampus. Mol Brain Res 42: 79-88, 1996.

4. Ruan YW, Ling GY, Zhang JL and Xu ZC: Apoptosis in the rat striatum after transient forebrain ischemia and the effects of ischemic severity. Brain Res 982: 228-240, 2003.

5. Liu C, Li J, Meng FY, et al: Polysaccharides from the root of Angelica sinensis promotes hematopoiesis and thrombopoiesis through the PI3K/AKT pathway. BMC Complement Altern Med 10: 79, 2010 .

6. Luo Y, Zhao HP, Zhang J, et al: Effect of ferulic acid on learning and memory impairments of vascular dementia rats and its mechanism of action. Yao Xue Xue Bao 47: 256-260, 2012 (In Chinese).

7. YiL, Liang Y, Wu H and Yuan D: The analysis of Radix Angelicae Sinensis (Danggui). J Chromatogr A 1216: 1991-2001, 2009.

8. Ma ZC, Hong Q, Wang YG, et al: Effects of ferulic acid on hematopoietic cell recovery in whole-body gamma irradiated mice. Int J Radiat Biol 87: 499-505, 2011.

9. Yan S, Xie Y, Zhu B, Han Y and Guo W: Effect comparison of different formulation of Dang-Gui-Bu-Xu-Tang on myelosuppression mouse. Asian Pac J Trop Med 4: 556-559, 2011.

10. Cheng CY, Su SY, Tang NY, et al: Ferulic acid provides neuroprotection against oxidative stress-related apoptosis after cerebral ischemia/reperfusion injury by inhibiting ICAM-1 mRNA expression in rats. Brain Res 1209: 136-150, 2008.
11. van der Kooij MA, Groenendaal F, Kavelaars A, Heijnen CJ and van Bel F: Neuroprotective properties and mechanisms of erythropoietin in in vitro and in vivo experimental models for hypoxia/ischemia. Brain Res Rev 59: 22-33, 2008.

12. England TJ, Gibson CL and Bath PM: Granulocyte-colony stimulating factor in experimental stroke and its effects on infarct size and functional outcome: A systematic review. Brain Res Rev 62: 71-82, 2009.

13. Yamada M, Burke C, Colditz P, Johnson DW and Gobe GC: Erythropoietin protects against apoptosis and increases expression of non-neuronal cell markers in the hypoxia-injured developing brain. J Pathol 224: 101-109, 2011.

14. Prass K, Scharff A, Ruscher K, et al: Hypoxia-induced stroke tolerance in the mouse is mediated by erythropoietin. Stroke 34: 1981-1986, 2003.

15. Sevimli S, Diederich K, Strecker JK, et al: Endogenous brain protection by granulocyte-colony stimulating factor after ischemic stroke. Exp Neurol 217: 328-335, 2009.

16. Jiang W, Fu F, Tian J, Zhu H and Hou J: Curculigoside A attenuates experimental cerebral ischemia injury in vitro and vivo. Neuroscience 192: 572-579, 2011.

17. Minematsu, Li L, Sotak CH, Davis MA and Fisher M: Reversible focal ischemic injury demonstrated by diffusion-weighted magnetic resonance imaging in rats. Stroke 23: 1304-1310, 1992.

18. Kirino T: Delayed neuronal death in the gerbil hippocampus following ischemia. Brain Res 239: 57-69, 1982.

19. Pulsinelli WA, Brierley JB and Plum F: Temporal profile of neuronal damage in a model of transient forebrain ischemia. Ann Neurol 11: 491-498, 1982

20. Noguchi CT, Asavaritikrai P, Teng R and Jia Y: Role of erythropoietin in the brain. Crit Rev Oncol Hematol 64: 159-171, 2007.

21. Wu H, Liu X, Jaenisch R and Lodish HF: Generation of committed erythroid BFU-E and CFU-E progenitors does not require erythropoietin or the erythropoietin receptor. Cell 83: 59-67, 1995.

22. Tsai PT, Ohab JJ, Kertesz N, et al: A critical role of erythropoietin receptor in neurogenesis and post-stroke recovery. J Neurosci 26: 1269-1274, 2006.

23. Chikuma M, Masuda S, Kobayashi T, Nagao M and Sasaki R: Tissue-specific regulation of erythropoietin production in the murine kidney, brain, and uterus. Am J Physiol Endocrinol Metab 279: E1242-E1248, 2000.

24. Yang Y and Rosenberg GA: Blood-brain barrier breakdown in acute and chronic cerebrovascular disease. Stroke 42: 3323-3328, 2011.

25. Sugiyama Y, Yagita Y, Oyama N, et al: Granulocyte colonystimulating factor enhances arteriogenesis and ameliorates cerebral damage in a mouse model of ischemic stroke. Stroke 42: 770-775, 2011.

26. Floel A, Warnecke T, Duning T, et al: Granulocyte-colony stimulating factor (G-CSF) in stroke patients with concomitant vascular disease--a randomized controlled trial. PLoS One 6: e19767, 2011.

27. Gibson CL, Bath PM and Murphy SP: G-CSF administration is neuroprotective following transient cerebral ischemia even in the absence of a functional NOS-2 gene. J Cereb Blood Flow Metab 30: 739-743, 2010. 